\title{
EL DERECHO AMBIENTAL DESDE UNA PERSPECTIVA DE DERECHOS HUMANOS Y DE EFICAZ GESTIÓN GUBERNAMENTAL
}

Dra. Romina Caminada.

\begin{abstract}
Resumen
En el presente artículo se desarrolla el tema del derecho ambiental, rama que con el transcurso del tiempo ha ido ganando importancia en las agendas gubernamentales dado a la problemática ambiental actual que se va agravando día con día. Por tales motivos, es necesario que se desarrolle una política ambiental sustentada sobre los pilares de aspectos económicos, políticos, sociales, culturales y ecológicos. Asimismo, la autora desarrolla los componentes a tomar en consideración para la obtención exitosa de objetivos claros en el plano ambiental. Finalmente, sintetiza las bases sobre el éxito de una política ambiental.
\end{abstract}

\section{Summary}

This article develops the subject of environmental law; a branch ihat over time has been gaining prominence on government's agendas because of the environmental degradation. For these reasons it is necessary to develop an environmental policv supported on the pillars of economics, politics, social, cultural and ecological themes. The author develops the components for obtaining a successfully results. Finally, she summarizes the requirements of a successful environmental policv.

\section{Sommaire}

Cet article développe le sujet de droit de I environnement; une branche qu au fil du temps a gagné en importance dans les agendas gouvemement en raison de la degradation de I 'environnement. Pour ces raisons, il est nécessaire de développer une politique environnementale en appui sur les piliers de I 'économie, la politique, des themes sociaux, culturéis et écologiques. L 'auteur développe les composants pour obtenir un résultat avec succés. Enfin, elle résume les exigences d'une politique environnementale efficace. 


\section{INTRODUCCIÓN}

El derecho ambiental es una rama del derecho vasto en su contenido y aplicación. Son muchas las áreas del derecho que afectan y cumplen roles para la aplicación del mismo. Más aún son muchas las carreras profesionales que deben aunarse a la creación de normas ambientales, a su regulación, entendimiento, aplicación y realización conjunta de cada uno de los objetivos del derecho ambiental.

Ramas del derecho como el derecho comercial, el derecho laboral, derecho minero, derecho a la propiedad intelectual y variadas profesiones ubicadas en el área de las ciencias, como la biología, la geología, la meteorología, la hidrología, la botánica, la bioquímica, etc., así como la intervención de otro tipo de profesiones que responden a una naturaleza tecnológica, como la ingeniería industrial, forestal, agropecuaria, alimentaria, etc., son instrumentos de aplicación necesarios en la búsqueda del cumplimiento de un reto, el cual consiste en la correcta implementación del derecho ambiental siguiendo lineamientos de eficacia y eficiencia enmarcados en términos reales y posibles que permitan una mejora en nuestro medio ambiente y por lo tanto en la vida y salud de cada uno de los ciudadanos que dependen de la realidad ambiental nacional y mundial.

Todos estos esfuerzos en el área ambiental necesitan, como ya hemos señalado, de diferentes ramas profesionales y éstas de diferentes ramas del derecho, siendo obviamente la del derecho ambiental la especialidad donde recaen las labores de sincronización, toma de decisiones, recolección y final empoderamiento de la información perteneciente a las competencias de otras áreas, de modo tal que la suma de conocimientos servirá como base para la orientación de políticas ambientales.

Hoy en día la problemática ambiental es finalmente una variable real de consideración por parte de los gobiernos. Sin embargo, el camino ha sido largo y no tan fácil. El derecho ambiental ha ido poco a poco llegando a las agendas nacionales. El proceso de toma de conciencia se dilató en el tiempo pero finalmente forma parte de las preocupaciones de nuestros políticos y de nuestros gobiernos.

Los inicios del derecho ambiental datan de finales del S. XIX y con la creación de organizaciones internacionales como las Naciones Unidas en 1945, llegando a su primer reconocimiento internacional con la consagrada Declaración de Estocolmo de 1972. ${ }^{154}$ Hasta ese momento la preocupación por el medio ambiente y el inicio de un derecho internacional ambiental se había plasmado en un documento internacional de rango simplemente declarativo; Declaración que consistía en la redacción de 26 principios, los que serían los nuevos estándares y referentes internacionales a los que cualquier gobierno firmante podía acudir como referente para la aplicación de los mismos como sus parámetros ambientales. Estos principios de carácter simplemente declarativo tenían grandes aspiraciones, las cuales consistían en su incorporación en alguna legislación, lo cual dependía de la voluntad política. Es decir, que si bien es cierto algunos gobiernos suscribieron aquellos principios reconocidos por la declaración de Estocolmo, todo esto se hizo bajo un marco internacional y referencial; por ello el verse finalmente incorporadas en legislaciones nacionales haría de estos principios legislación oponible a los ciudadanos del gobierno que decidiese optar por la incorporación nacional de los mismos, dejando de lado la calidad simplemente declarativa para convertirse en ley nacional.

154 Aguilar, Grethel e Iza, Alejandro. "Manual de Derecho Ambiental para Centroamérica*'. Centro de Derecho Ambiental de la Unión Mundial para la Naturaleza. Oficina Regional para Mesoamérica. 2005. p 25-28. Disponible en: http://data.iucn.org/dbtw-vvpd/edocs/ELC-017.pdf 
El Derecho ambiental desde una Perspectiva de Derechos - 283

EIUMANOS Y DE EFICAZ GeSTiÓn GUBERNAMENTAL

Antes de llegar a estas incorporaciones legislativas o constitucionales, las declaraciones son simplemente lo que su palabra señala declaraciones de buena voluntad de los Estados Partes, más no compromisos vinculantes. Por ello la importancia vital de su incorporación formal en la legislación nacional.

Siguiendo la línea de desarrollo y avance del derecho ambiental en el contexto internacional, tenemos que de modo posterior a la Declaración de Estocolmo se presenta como un segundo hito de desarrollo, en el año de 1987, el Informe "Nuestro Futuro Común", más conocido como el "Informe Brundtland", el cual da a luz al término "desarrollo sostenible". El concepto de desarrollo sostenible queda definido como "el desarrollo que permite la satisfacción de las necesidades presentes sin poner en peligro la capacidad de las generaciones futuras para satisfacer sus propias necesidades". 155

Por otro lado el trabajo del Panel Intergubernamental de Cambio Climático creado en 1988, fue vital para el posicionamiento del tema. El primer informe de evaluación del Panel Intergubernamental de Cambio Climático entregado en 1990, dio lugar a la iniciativa de las Naciones Unidas de la Convención Marco sobre Cambio Climático. 156

En 1992, la Asamblea General de las Naciones Unidas acordó realizar la Conferencia sobre Medio Ambiente y Desarrollo en Río de Janeiro. "Pensar globalmente, actuar localmente", fue el lema de la Cumbre de Río, mediante la cual se acordó presentar a los Estados, los objetivos de una alianza mundial bajo un plan de acción para el desarrollo sostenible mundial. 157

La Cumbre de la Tierra congregó a los Jefes de Estado más importantes y se adoptaron cinco instrumentos internacionales de gran relevancia: la Declaración de Río de Janeiro sobre Medio Ambiente y Desarrollo, el Programa o Agenda 21, la Declaración sobre bosques, la Convención de Naciones Unidas sobre Diversidad Biológica y la Convención Marco de las Naciones Unidas sobre Cambio Climático. ${ }^{158}$

Dentro de los instrumentos producidos en el Marco de la Cumbre de la Tierra, la importancia de la Agenda 21 en el desarrollo del derecho ambiental internacional tiene un matiz diferencial y éste se debe a su perspectiva de aplicación.

La Agenda 21 señala que la acción local no sustituye a la acción nacional ni internacional, más bien, las complementa. Los dos pilares sobre los que se asienta esta Agenda, son por un lado los principios de gestión ambiental y por otro lado, la toma democrática de decisiones, analizando ambos principios bajo el concepto de sostenibilidad, lo cual implica el mantenimiento de la biodiversidad, la salud humana, la calidad del agua, del aire y del suelo para mantener la vida humana, animal y vegetal. 159

\footnotetext{
155 Vera Esquivel, Germán. "Introducción al Derecho Internacional del Medio Ambiente". ARA Editores. Primera Edición 2011. p. 102 citando a Comisión Mundial del Medio Ambiente y del Desarrollo "Nuestro Futuro Común". Bogotá, Alianza Editorial colombiana. Colegio Verde de Villa de Leyva, 1988. p 67

156 Galarza. Elsa. Los costos económicos del cambio climático. Economía ySociedad 67. CIES. Abril 2008 p. 15

157 Vera Esquivel, Germán, Op. cit., p. 107.

$158 \quad$ Vera Esquivel, Op. cit. p. 108.

159 Flores Marbán, Raquel. "La Agenda 21 impulsora del desarrollo sostenible y de la protección del medio ambiente en Europa y España". Boletín Económico de ICE No 2899. Del 11 al 17 de Diciembre de 2006. Disponible en: http://www.revistasice.com/CachePDF/BICE_2899_31-46 8E1D85309A45454 E09932F953D53CF8E.pdf
} 
Es gracias a este documento internacional que los temas ambientales comienzan a ser entendidos y abordados desde una perspectiva de acción local.

Por otro lado, en el año 2002, el objetivo de la Cumbre de Johannesburgo, estuvo centrado en la obtención de dos tipos de resultados, por un lado una "Declaración Política" y un "Plan de Aplicación", y por otro lado la formación de alianzas para el desarrollo sostenible, las que encontrarían su sustento en las primeras.1611

Ya a partir del año 2002 se puede decir que el tema del cambio climático es el que ha encabezado la agenda ambiental internacional, destacándose como señala Vera Esquivel, el informe Stem, las opiniones de Al Gore y la Cumbre de Copenhague sobre Cambio Climático. ${ }^{161}$

A pesar de los primeros esfuerzos ya referidos por parte del Panel Intergubernamental de Cambio Climático, es recién con el segundo informe del 2005 que se mostró mayor evidencia científica de los cambios en el clima de la tierra, sus impactos y futuras consecuencias y se proporcionó material para las negociaciones del Protocolo de Kioto. ${ }^{162}$ Es así que con la entrega de este segundo informe, por parte del Panel Intergubernamental de Cambio Climático, el tema del cambio climático dejó de ser solo un asunto de científicos y empezó a entrar en las agendas políticas de diferentes países. ${ }^{163}$

El año 2005, debe ser considerado como un hito importante para el tema ambiental y por consiguiente del derecho ambiental, ya que una vez captada la atención y preocupación de la comunidad internacional es cuando, finalmente se logra elevar tal inquietud al plano de acción de los gobiernos, y por lo tanto del uso del derecho, como mecanismo asertivo de control y posible prevención de uso correcto y saludable de estándares ambientales indicados.

Como podemos apreciar es una lucha de más de 60 años, de un progreso escalonado y paulatino, relacionado con la incorporación de los temas ambientales al derecho internacional y a las agendas nacionales. Ha sido también una lucha del derecho ambiental para llegar a su reconocimiento como mecanismo orientador, protector y regulador de los temas ambientales y la aceptación de tales cuestiones como problemáticas de alta consideración por parte de los Estados. Desgraciadamente la llegada del derecho ambiental en el marco internacional y, más aún, en los marcos regionales y nacionales, llega con posterioridad a los problemas ambientales. Siendo catalogada por algunos autores como Orlando Rey como una llegada tardía ${ }^{164}$. Razón por la cual, se anhela que principios como el principio de prevención o el principio preventivo empiecen finalmente a obtener un mayor peso de aplicación en nuestros días, para que de algún modo el derecho ambiental comience a llegar con antelación a algunos problemas ambientales.

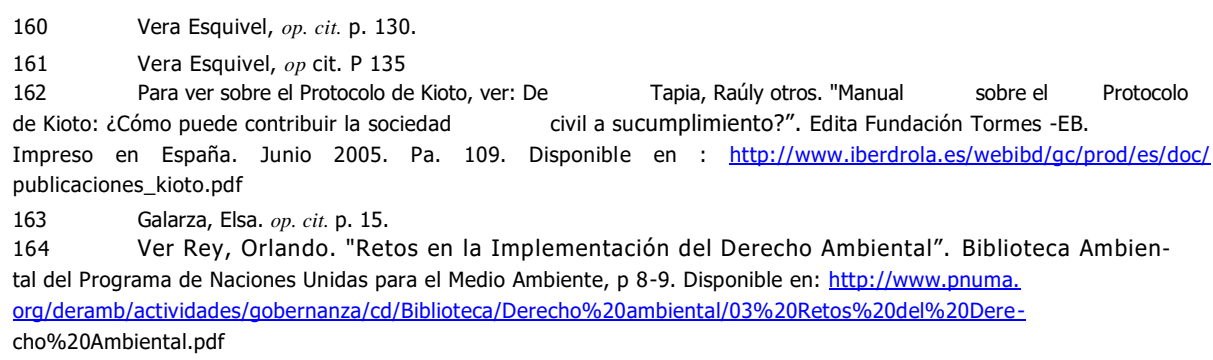


El Derecho ambiental desde una Perspectiva de Derechos - 285

Humanos y DE Eficaz Gestión GUBeRnamental

Así tenemos que, un principio de prevención nos señala que la gestión ambiental tiene como objetivos el prevenir, vigilar y evitar la degradación ambiental; y cuando las causas que la generen no sean posibles de ser eliminadas, se adopten otro tipo de medidas, como las de mitigación, recuperación, restauración o compensación según corresponda. ${ }^{165}$

Por otro lado, el principio precautorio nos señala que cuando exista un peligro de daño grave e irreversible, es decir un tipo de daño que contenga ambas características, la falta de certeza científica no podrá utilizarse como razón para postergar la adopción de medidas eficaces y eficientes para impedir la degradación del medio ambiente. ${ }^{166}$

La regularidad en la aplicación de estos dos principios, es en la actualidad un nuevo reto a abordar por parte del derecho ambiental. Es decir, que el derecho ambiental empiece a tener una llegada a priori a los futuros problemas ambientales o a las futuras y no por ello lejanas consecuencias ambientales, producto de problemas actuales de naturaleza medio ambiental; y así lograr la aplicación y la consolidación de aquella naturaleza preventiva que es parte fundamental de este derecho ambiental.

A todo lo expuesto, es importante decir que el éxito del desarrollo ambiental reflejado en la conservación de nuestro medio ambiente a nivel mundial, no sólo depende del desarrollo progresivo de los estándares internacionales, o de las incorporaciones constitucionales del tema, o de las producciones legislativas, o de la creación de ministerios e instituciones dirigidos a la protección del mismo, sino de la suma de todas estas medidas y demás formas de aplicación de las mismas, sinergia que explicaremos a continuación.

\section{COMPONENTES A TOMAR EN CONSIDERACIÓN PARA LA OBTENCIÓN EXITOSA DE OBJETIVOS CLAROS EN EL PLANO AMBIENTAL:}

La existencia de una voluntad política por parte de los gobiernos, el interés de nuestros diferentes partidos políticos y el estudio que realicen agencias de organismos internacionales, como son los realizados por las Naciones Unidas mediante por ejemplo el Programa de Naciones Unidas para el Medio Ambiente, configuran elementos de base, de impulso e incentivo de conciencia ambiental. Sin embargo, todo este tipo de esfuerzos, llámese voluntad política $y / 0$ incentivos políticos internacionales, carecería de todo real significado de aplicación triunfante dentro de las variantes destinadas a la creación de una fórmula de éxito de los temas ambientales y por ende del derecho ambiental si es que no llega a entenderse que otras variables son más que necesarias para la obtención de tales logros comunes.

Estas Ilamadas variables adicionales y no por ello secundarias, responden a otro tipo de labor por emprender. Nos referimos a la educación y cultura ambiental, a las campañas de sensibilización, de información, no por gusto uno de los principios rectores del derecho ambiental son el derecho de información y el derecho de participación ciudadana .La aplicación de esos principios tiene un peso clave dentro de los retos de implementación y aplicación del derecho ambiental.

165 C'arhuatocto Sandoval, Henry. "Los principios del Derecho Ambiental en la Ley General del Medio Ambiente", p. 16. Disponible en: http://xa.yimg.com/kq/groups/15536342/2021945107/name/LOS +PRINCIPIOS+DEL+DERECHO+AM BIENTAL.pdf

166 Ibid, p. 19 
El conocimiento real de las problemáticas ambientales debe llegar a la ciudadanía, a través de diferentes medidas que pueden ser adoptadas a través de las distintas competencias que manejan cada ministerio o cada entidad regional, local o municipal. Hay que tomar más que en cuenta que la población es la que optará día a día por una aplicación de las medidas ambientales impuestas por ley y que desgraciadamente, muchas veces le resultan desconocidas. Esta falta de información no comulga con una política de fomento a un medio ambiente sano basado en el principio de sostenibilidad y. menos aún, en los principios de aplicación a priori ya mencionados.

De la existencia de medidas legislativas coherentes, así como la presencia de gobiernos que apliquen sus políticas de modo transversal, dependerá el éxito de protección del medio ambiente. Caso contrario, quedaría simplemente un listado de leyes que pretenden proteger el medio ambiente. La consideración de estos componentes de naturaleza diferente nos lleva a la pregunta: ¿cuál sería la receta para alcanzar el éxito en la protección del medio ambiente, en términos de que la aplicación del derecho ambiental resulte eficiente y eficaz?

La fórmula a diseñar es algo compleja y cada una de sus variables se descompone en diferentes matices de correcta aplicación. Aquí trataremos de esbozar tal fórmula, entendiendo que cada componente tiene una ramificación de elementos para su cabal entendimiento. Es así que para el éxito del derecho ambiental necesitamos de:

a) El reconocimiento del derecho ambiental como un derecho humano que es.

Esta afirmación que puede verse consolidada mediante el reconocimiento constitucional del derecho a un ambiente sano y a una calidad de vida por parte del ciudadano, derechos de los cuales María del Carmen Carmona ${ }^{1,7}$ nos habla abiertamente en su artículo "Derechos Humanos y Medio Ambiente", y que son puntos que nos deja en claro la esencia del derecho ambiental y de su calidad como derecho humano ${ }^{168}$. Un derecho humano de tercera generación, reflejado en un derecho al Medio Ambiente, y un derecho al Desarrollo, derechos cuyos logros de objetivos dependen de la colectividad y no solo de un individuo. El alcance de sus metas depende de comunidades, de la comunidad internacional y de la clara intervención de los Estados y sus gobiernos.

Si no logramos el reconocimiento del derecho ambiental, al estándar de un derecho a un medio ambiente sano, de un derecho a una calidad de vida, a la categoría de derecho humano, restamos importancia al mismo, quitamos la real perspectiva que el tema tiene y dejamos de sintonizar el objetivo que merece el desarrollo sostenible, el que se relaciona con la vida de las presentes generaciones y de las futuras generaciones.

El derecho ambiental siempre estará relacionado con los derechos humanos de todas las personas y con mayor énfasis de grupos vulnerables como lo son los pueblos indígenas y las poblaciones que viven en extrema pobreza.

167 Ver Carmona Lara, María del Carmen. "Derechos Humanos y Medio Ambiente". Artículo que

forma parte de la biblioteca Jurídica Virtual del Instituto de Investigaciones Jurídicas de la Universidad Nacional Autónoma de México UNAM. p.5, 13-17. Disponible en: http://biblio.juridicas.unam.mx/libros/6/2759/4.pdf.

$168 \quad$ Nuestra actual Constitución Política de 1993 acoge este derecho a través de su artículo 2.11, al

señalar "Toda persona tiene derecho de toda persona a la paz, a la tranquilidad, al disfrute del tiempo libre y al descanso, así como a gozar de un ambiente equilibrado y adecuado al desarrollo de su vida. El tema ambiental sigue protegido en el Capítulo II "Del Ambiente y los Recursos Naturales", en sus artículos 66 al 
El Derecho ambiental desde una Perspectiva de Derechos $\mathbf{- 2 8 7}$ humanos y de EFicaz Gestión Gubernamental

Y ¿por qué decimos algo así? La respuesta es muy fácil, el primer grupo en mención, los pueblos indígenas ${ }^{161} *$ corresponden a grupos poblaciones que aplican de mejor modo el principio de sostenibilidad del cual hablamos y rigen sus vidas de manera increíble con tal principio y las labores del derecho ambiental. Son los pueblos indígenas los que sin matricularse en cursos básicos ni avanzados de Derecho al Desarrollo Sostenible, los que hacen uso de los recursos naturales de modo inteligente y eficiente. Sus condiciones de vida, sus tradiciones, son un correlato viviente de una perfecta aplicación del principio de sostenibilidad. Ahora, en relación con el segundo grupo referido a las poblaciones que viven en extrema pobreza, tenemos que los efectos negativos de los desastres naturales afectan de manera directa a las poblaciones pobres ya que éstas son las que viven directamente de la naturaleza, de su materia prima, son los grupos poblacionales que tienen menores recursos para protegerse de los efectos que trae el cambio climático para la naturaleza. Es así que los desastres naturales siempre afectan a estos grupos vulnerables de manera trascendental. Con este último punto queda reafirmada la vinculación de los temas de derechos humanos con el derecho ambiental, no sólo por el derecho a una vida sana y de calidad que tiene todo ser humano, sino por la vinculación necesaria del derecho ambiental con los grupos vulnerables mencionados, quienes son materia de especial protección por los derechos humanos y que se encuentran en una estrecha y dinámica relación con el medio ambiente y sus problemáticas.

El concepto de calidad de vida, ligado directamente con el derecho a la vida, derecho humano por excelencia, empieza a jugar un rol preponderante en la aplicación de políticas medio ambientales, ya que como señala María del Carmen Carmona en el artículo ya referido, el vínculo existente entre los derechos humanos y los derechos ambientales se encuentra en la existencia de elementos que establecen la conexión entre ellos, los que responden a dos categorías, el elemento humano plasmado en un orden jurídico y al mismo tiempo en su efectividad para garantizar el respeto a los derechos humanos, y el elemento material que pone al desarrollo sustentable como requisito esencial para obtenerlo. ${ }^{170}$

b) Aplicación del principio de sostenibilidad como rector de toda política ambiental

Este derecho humano al Medio Ambiente y al Desarrollo ${ }^{171}$, implica la aplicación de un principio rector en el ámbito ambiental, el principio de sostenibilidad.

Este principio de sostenibilidad debe estar basado en un análisis económico, político, social y cultural y ambiental sobre la base de decisiones tomadas bajo consideraciones éticas. El eficiente uso de los recursos, tomando en cuenta la limitación de la pobreza y la equidad social, el respeto a las diversas culturas y la preservación del 
medio ambiente, sobre la base de políticas públicas e inversiones privadas éticas, es el objetivo de dinámica ambiental que busca el desarrollo sostenible 173.

En este sentido, resulta muy interesante la propuesta de Guimaráes, a partir de una imagen sugerida inicialmente por Otis Duncan, y citado por Carmona, cuando acertadamente señala, que la sustentabilidad de una comunidad va a residir de la interrelación entre los elementos: población, organización social, entorno, tecnología y aspiraciones sociales. ${ }^{173}$

Mientras Otis Duncan, citado por la misma Carmona, señala que la calidad de vida es la forma de determinación de la sustentabilidad de una comunidad en espacio y tiempo determinados y que dicha sustentabilidad va a depender del equilibrio dinámico de elementos claves como la población, la organización social, el entorno, el territorio, la tecnología aspiraciones sociales.

Así tenemos, que el primer elemento, referido a la población, nos va a permitir estudiar varios factores que dan índices de la calidad de vida, como son los aspectos demográficos, migratorios, ambientales, de control poblacional, etc. Dentro del análisis correspondiente a este elemento población, encontramos diferentes factores que pueden concluir en un mejor estudio del contexto bajo el cual se puede desarrollar una vida y al fin y acabo desarrollar una calidad de vida. ${ }^{174}$

Es ahí que nos damos cuenta que la transversalidad en el tema ambiental ocupa un sitio preponderante de aplicación, aspecto que abordaremos más adelante como otra variable necesaria para el alcance del éxito por parte de una política ambiental de protección al medio ambiente.

El tema poblacional tiene que ver con factores como la alimentación, el agua potable, los grupos étnicos, con el mismo medio ambiente, y la necesidad de adopción de políticas de protección de medio ambiental a favor de las poblaciones en riesgo. 175

Como podemos observar hasta el momento un gran porcentaje de los Objetivos del Milenio de las Naciones Unidas se encuentran inmersos en este análisis que estamos realizando. Es decir se encuentran presentes como factores en nuestras variantes. Entre los Objetivos del Milenio, comunes al estudio en mención, tenemos la erradicación de la pobreza extrema y el hambre, la reducción de la mortalidad infantil, el mejoramiento de la salud materna, así como la lucha por la garantía de la sostenibilidad del medio ambiente y el fomento de una asociación mundial para el desarrollo. 176

172 Barrerira, Ana; Ocampo, Paula; y Recio, Eugenia, Medio Ambiente y Derecho Internacional: Una Guía Práctica. Caja Madrid, e Instituto Internacional de Derecho y Medio Ambiente (IIDMA) Madrid 2007. p 35 y 36

Declaración de Rio sobre Medio Ambiente y Desarrollo, 1992. Disponible en: http://www.pnuma.org/ docamb/dr1992.php y

Declaración de Johannesburgo sobre el Desarrollo Sostenible en: UN Department of Economic and Social Affairs. División for Sustainable Development.. Desde nuestro origen hasta el futuro. Disponible en: http:// www.un.org/esa/sustdev/documents/WSSD POI PD/Spanish/WSSDsp_PD.htm

173 Carmona, María del Carmen, Op. Cit. p 9. Cita a: Guimaraes P., Roberto "La ética de la sustentabilidad y la formulación de políticas de desarrollo" Revista Ambiente y Sociedad, núm. 2, 1998 (Campiñas, Brazil), primer semestre, p. 5-24 a partir de ideas descritas por Ducan, Otis "From Social System to Ecosystem", Social Inquiry", Chicago, núm. 31, invierno, 1961, p. 140-149.

174 Carmona, María del Carmen, Op.Cil. p 9. Cita a: Ducan, Otis "From Social System to Ecosys tem", Social Inquiry", Chicago, núm. 31, invierno, 1961, p. 140-149.

175 Carmona, María del Carmen Op. Cit. p. 9-10.

176 Sistema de Naciones Unidas en el Perú. Disponible en: http://www.onu.org.pe/Publico/odm/odm.aspx 
El Derecho ambiental desde una Perspectiva de Derechos - 289 humanos y de Eficaz Gestión Gubernamental

Retomando el análisis de los elementos, tenemos que el tema de la organización social se relaciona con los derechos políticos vinculados con las democracias y las formas de gobierno, las formas de gestión del agua y del territorio. Estando al mismo tiempo vinculado con los derechos de participación, de asociación, dándole relevancia al derecho de información. Por otro lado el elemento entorno se relaciona directamente con el medio ambiente y todos los temas relacionados al mismo, las áreas naturales protegidas, la protección a la biodiversidad, el acceso a los recursos genéticos, la contaminación, los tóxicos. El elemento territorio tiene que ver con el ordenamiento territorial, con temas como la propiedad de las tierras, la planeación urbana. El elemento tecnología responde a temas de derecho, como la propiedad intelectual, las patentes, las marcas, el acceso a los recursos naturales o genéticos. Y finalmente, las aspiraciones sociales que abarcan las metas que la sociedad desea plantearse como tal, entre ellas, el mejoramiento ambiental, la calidad de vida, el bienestar, el desarrollo, etc. 177

\section{c) Otra variable a considerar es el entendimiento de un marco jurídico $\boldsymbol{u}$} ordenamiento sólido'.

El respeto a la palabra empeñada bajo los diferentes compromisos adoptados por medio de los tratados internacionales ratificados por el gobierno así como el trabajo de un marco legal coherente que no permita la yuxtaposición de las normas, el desorden jurídico y la introducción de normas cuya redacción jurídica esté erróneamente basada en el alcance de objetivos utópicos y/o imposibles sociales, mediante la idealización de metas alejadas de una realidad económica, social y política y otras veces simplemente contradictorias entre sí. 178

El trabajo de manejar un marco legal coherente, es una labor ardua que necesita del estudio y la continua revisión de normas antiguas ya expiradas o reemplazadas por nuevas para así lograr una fluidez del cuerpo legal nacional bajo una misma concepción de respeto al derecho ambiental.

\section{d) Implementación del Poder Judicial:}

Del mismo modo la implementación legal no llegaría a ningún rumbo de escaso éxito del derecho ambiental y por lo tanto del respeto al medio ambiente, si el poder judicial, aquel poder del Estado que dentro de un Estado democrático es el ente encargado de la aplicación e interpretación de las normas, no se encuentra actualizado y entrenado en esta área del derecho. Por ello la importancia de la capacitación de este poder del Estado, en esta rama del derecho que es relativamente contemporánea.

e) Transversalidad en el diseño y aplicación de las políticas ambientales:

Otra variante extremadamente vital para la evaluación de buenos resultados, es el tema de la gestión gubernamental de modo transversal, la cual recae de modo especial en el Poder Ejecutivo, en cada uno de los Ministerios que conforman este poder del Estado. Es así que la política medio ambiental, los parámetros, guías, enfoques, soportes y aplicación de un Plan Nacional de Medio Ambiente, del cuerpo jurídico que respalda las medidas ambientales, o de la aplicación de los convenios internacionales suscritos, va a 
depender en gran medida de un trabajo transversal más que necesario de cada norma ambiental.

No podemos entender por ejemplo que la aplicación de un Plan Nacional de Medio Ambiente dependa sólo de un Ministerio del Ambiente, no podemos entender que una política de Estado alcanzará de modo responsable y sensato la implementación de una política ambiental sin que exista un trabajo de análisis transversal de los temas y de final reconciliación de perspectivas en las medidas que se adopten.

Es así que debemos entender que muchas de las carteras ministeriales se encuentran altamente relacionadas con los objetivos de un Ministerio del Ambiente. Así tenemos al Ministerio de Agricultura, el Ministerio de Energía y Minas, el Ministerio de Economía y Finanzas, el Ministerio de la Producción, el Ministerio de Comercio Exterior y Turismo, el Ministerio de Salud, el Ministerio de Transporte y Comunicaciones, entre otros y tal vez menores niveles de relación, pero de ningún modo mínimos y menos aún nulos, el Ministerio de Educación y el Ministerio de la Cultura, por ejemplo.

No podría existir una política real de medio ambiente por parte de un gobierno si cada Ministerio trabaja de modo aislado para la obtención de objetivos nacionales medio ambientales. Más aún es ingenuo pensar que el tema ambiental sea un tema de objetivos cuyo espectro de acción sólo depende de la activación de las competencias de un Ministerio del Ambiente, o peor aún, pensar que esas competencias son autónomas, sin vínculo alguno a otros ministerios, instituciones o gobiernos locales o municipales. Pensar de este modo desembocaría en resultados contradictorios, poco entendibles, y en el mejor de los casos escasamente exitosos. Resultados que se verían reflejados, por ejemplo, en que temas ambientales resulten teniendo distintas lecturas por parte de algunos de los ministerios involucrados en tales problemáticas, derivando en una disputa fáctica de competencias ministeriales, o en un ir y venir de producciones legislativas.

El manejo transversal de las carteras ministeriales es una tarea difícil, ardua y hasta titánica. Significa y se traduce en remisión de documentos, constante comunicación entre las partes involucradas, voluntad de entendimiento y de negociación. No es fácil, pero es el único modo de trabajar para el presente y para el futuro. Es el modo que permitirá el alcance de políticas consolidadas, comprensibles y posibles; evitando que los programas o políticas medio ambientales terminen siendo parches casuales $y$ contemporáneos, capaces de sufrir mutaciones dependiendo de los gobiernos y autoridades en funciones.

Así tenemos que un Ministerio de Comercio Exterior y Turismo es fundamental para la recepción e ingreso paulatino de una economía verde en un país. Una economía verde es una opción de política económica que lleva como base el respeto al medio ambiente y la aplicación del principio de sostenibilidad mediante la implementación de medidas comerciales, económicas, laborales que no transgredan el medio ambiente y que en pocas palabras apliquen el concepto de sostenibilidad en gran medida, donde el uso eficiente de los recursos es la línea de conducción de las medidas a adoptar, donde ese uso eficiente e inteligente tenga como meta el razonamiento entendido que los recursos naturales no sólo pertenecen a la generación presente sino también a las futuras generaciones. 
El Derecho ambiental desde una Perspectiva de Derechos - 291 Humanos y de EEICAZ GESTión Gubernamental

"El Programa de Naciones Unidas para el Medio Ambiente considera que una economía verde debe mejorar el bienestar del ser humano y la equidad social, a la que vez que reduce significativamente los riegos ambientales y la escasez ecológica. En su forma más básica una economía verde sería aquella que tiene bajas emisiones de carbono, utiliza los recursos de forma eficiente y socialmente incluyente. En una economía verde el aumento de los ingresos y la creación de empleos deben derivarse de inversiones públicas y privadas destinadas a reducir las emisiones de carbono y la contaminación, a promover la eficiencia energética así como el uso de los recursos, y a evitar la pérdida de la diversidad biológica y de servicios de los ecosistemas. Dichas inversiones han de catalizarse y respaldarse con gasto público selectivo, reformas políticas y cambios en la regulación. El camino hacia el desarrollo debe mantener, mejorar y. donde sea necesario, reconstruir el capital natural como activo económico fundamental y fuente de beneficios públicos, especialmente para las personas desfavorecidas suyo sustento y seguridad dependen de la naturaleza". 179

Esta política de economía verde, permite tomar en consideración variante nuevas, como lo son el tema pobreza, el tema de inclusión social, y el tema de desarrollo. Como señala el Informe del Programa de Naciones para el Medio Ambiente, se necesita tomar muchos pasos adelante y preparar el camino de transición de una economía marrón a una economía verde, lo que el informe identifica como circunstancias favorables específicas, las que consisten en normativas, políticas, subsidios e incentivos nacionales sin dejar de lado obviamente el marco referencial internacional como lo es el mercado internacional y los protocolos de comercio. 180

Son muchos los factores que dificultan la transición hacia una economía verde. Transformación que implicaría el enverdecimiento progresivo de las diferentes carteras ministeriales a partir de la adopción de normativas competencia de sus carteras. como los mencionados subsidios e incentivos nacionales, o la alza de aranceles a determinados productos no ecológicos, competencias que corresponderían abordar a un Ministerio de Comercio Exterior y Turismo, el cual podría implementar medidas responsables que reflejen el apoyo a una economía verde, mediante la dación de aranceles que castiguen aquellos productos que dañen el medio ambiente. Es decir, adoptar un pensamiento de defensa a nuestro medio ambiente ante alguna firma de tratado internacional en materia comercial que la pueda perjudicar. Así como medidas comerciales incentivadoras de consumo de productos ecológicos. Medidas que pueden adoptarse en marcos regionales e internacionales.

De manera sincronizada a la adopción de estas medidas, el Ministerio de Agricultura podría intervenir con políticas referidas al agro que frenen o desincentiven el consumo de pesticidas u otros elementos básicos para las cosechas, que sean productos que por su composición se opongan a una salud alimentaria o que puedan significar un gran peligro para nuestra biodiversidad. Las políticas agrarias deben ir de la mano con lo señalado por nuestra Política Nacional de Medio Ambiente, respetando la ratificación de convenios internacionales como el Convenio de Biodiversidad, ateniéndonos al respeto e incentivo de nuestra biodiversidad, y al entendimiento que esta debería ser nuestra característica primordial como país.

179 Informe "Hacia una Economía Verde" del Programa de Naciones Unidas para el Medio Am-

biente. p. 01-02 (01) Disponible en : http://www.unep.org/greeneconomy/Portals/88/documents/ger/GER synthesis_sp.pdf

180 Loe. $\mathrm{Cu}$. p. 01-02(01).

athIna 
El trabajo realizado por algunos privados en el cultivo y fomento de productos orgánicos, es una prueba de ello. Si los privados han trabajado y apostado por ello, con políticas empresariales de mediano plazo, logrando que campesinos puedan hoy en día vivir del cultivo de tales productos mediante la comercialización de los mismos a empresas privadas peruanas, quedando claro que las cosas se pueden hacer bien. $Y$ es así, que si ha existido la inversión privada para la generación de estos puestos de trabajo verde, y este ciclo de producción, comercio y consumo enverdecido y exitoso, es entonces más que posible y necesario, que la inversión pública pueda bajo los mismos parámetros sostenibles, y por ello, social, cultural, político, económico y ecológicamente responsable, aplicar esta misma ruta triunfante en distintos sectores viables a ser enverdecidos.

Parte de las políticas planteadas en el discurso de nuestro Ex Primer Ministro Salomón Lemer, del 25 de Agosto de 2011 ante el Congreso de la República ${ }^{181}$, resultan asertivas, teniendo como objetivo la inclusión productiva al mercado de productores campesinos que viven en condiciones de subsistencia, usando herramientas políticas como la existencia de un agrobanco, la implementación de proyectos de irrigación y la idea de un censo agropecuario, puntos que toman en cuenta el aspecto social, el tema del territorio, uso eficiente de tierras y activación económica social de un sector tan importante de nuestra economía que es al igual que el sector ganadero y la pesca, son fuentes de trabajo de un gran porcentaje de nuestra población y al mismo tiempo son sectores cuyo dirección representarán el respeto a una política ambiental responsable.

Otros ejemplos de transversalidad aplicada en un poder ejecutivo, se encuentran en la implementación de medidas preventivas de contaminación del medioambiental a través de un Ministerio de Transporte y Comunicaciones que no puede ser ajeno a una política de medio ambiente, cuando son los automóviles grandes contaminantes de nuestras ciudades. Así tendríamos la intervención de un Ministerio de Transporte y Comunicaciones en la adopción de medidas pro-ambientales como la restricción a la circulación de vehículos terrestres de gran antigüedad, ya que los motores pueden perjudicar gravemente nuestro medio ambiente tomando en consideración el alto grado de emisión de carbono que producen.

Asimismo, un Ministerio de Vivienda, Construcción y Saneamiento que podría trabajar en la elevación de estándares nacionales en temas de urbanización y construcción. Además, debería abordar con mayor rigurosidad el previo estudio de cada uno de sus proyectos a desarrollar. Es así que existen muchos temas desarrollados por esta cartera que se relacionan con la problemática ambiental. Para mencionar uno de ellos tenemos la construcción de carreteras. Las carreteras son vitales para el desarrollo de las poblaciones en diferentes temas como el comercio, es por ello que este Ministerio no puede ser ajeno a tal problemática y debe tomar en cuenta la finalidad y objetivo en la construcción de carreteras, teniendo que tomar en consideración el análisis de las poblaciones favorecidas por estos proyectos, formulándose preguntas como, ¿A qué pueblos son los que finalmente conectarían estas carreteras?, ¿Quiénes harán uso de las mismas? ¿Reduciría los gastos de tales poblaciones? ¿Tal proyecto dejaría de encarecer los productos de venta de las poblaciones vulnerables? 
¿Permitiría a tales poblaciones obtener precios más competitivos, con lo que incentivaría un mayor consumo de sus productos o materia prima objeto de comercio? Lo que tal vez finalmente les permitiría a estos grupos poblacionales tener una esperanza concreta para salir de la extrema pobreza. Siempre entendiendo que todas estas interrogantes son componentes de esa dinámica analizada de calidad de vida vinculado directamente al tema ambiental.

Un ingreso acompasado de los considerandos señalados lleva consigo la implementación de una economía verde, un trabajo que debe verse impulsado en todos los estratos del gobierno.

Esta transversalidad reconocida por el Política Nacional de Medio Ambiente no sólo pasa por su aplicaron a nivel de Poder Ejecutivo, sino en todos los niveles de poder, desde el gobierno nacional, pasando por los gobiernos regionales, locales y municipales. Grandes directivas y pequeñas implementaciones bajo un misma línea rectora es a lo que debe estar dirigida una responsable política ambiental que aspire tener un real abordaje de la problemática ambiental nacional.

El informe sobre Economía Verde del Programa de Naciones Unidas para el Medio Ambiente titulado, "Hacia una Economía Verde" demuestra que el enverdecimiento de la economías no debe ser un problema para el crecimiento sino un nuevo incentivo del crecimiento, pudiendo ser considerada tal vez, como una nueva táctica para erradicar la pobreza, a través de los intentos de motivación a aquellas personas encargadas de la formulación de políticas y también mediante la creación de las condiciones propicias, ya antes referidas, para que de este modo se pueda incentivar una mayor inversión. Como bien señala el informe, la motivación hacia este incentivo de inversión se puede encontrar en una base de argumentos económicos, que buscan sustentar un cambio en la inversión tanto pública como privada, permitiendo de este modo, el hallazgo acertado de sectores claves a ser enverdecidos. Este informe pretende mostrar como una economía verde puede reducir la pobreza a partir del fomento de un empleo en sectores claves y basados en la aplicación del principio de sostenibilidad, como la agricultura, la ganadería, la pesca, la silvicultura, concluyendo finalmente en las políticas dirigidas a la reducción o eliminación de subsidios perjudiciales para el medio ambiente. 182

Obviamente la responsabilidad de carteras tan ligadas a problemáticas ambientales como el Ministerio de Energía y Minas tiene una tarea titánica de buscar el punto justo de encuentro, equilibrio y reconciliación entre una política extractiva y una política ambiental y socialmente responsable.

Las consideraciones ambientales están ligadas a la extracción y al uso eficiente e inteligente de mucho de nuestros recursos naturales, es decir el fomento de una política extractiva con bases de responsabilidad social y ambiental ya mencionadas, desarrollada ésta última bajo el principio de desarrollo sostenible. El compromiso señalado en el discurso del 26 de agosto de 2011, de una transformación de la actual matriz energética nacional dependiente de los hidrocarburos a una donde los recursos renovables tengan mayor participación es un ejemplo de ello. El desarrollo de proyectos energéticos a mediano y largo plazo, la determinación que las futuras concesiones sobre utilización de los recursos naturales renovables serán a plazo determinado, la construcción de hidroeléctricas, el desarrollo de redes de energías más económicas y ambientalmente pertinentes son aspectos positivos a considerar. 
e) Una variante final y no por ello menos importante es la existencia de una base democrática de respeto a las instituciones y canales democráticos.

El enfoque que corresponde plantear a una política pública sana debe responder a bases de responsabilidad social bajo la utilización de instrumentos económicos provenientes de una economía de mercado responsable y un estado de derecho que está caracterizado por un régimen democrático reflejado en la separación de poderes e institucionalidad con una garantía de respeto a los derechos humanos.

\section{CONCLUSIONES}

Debemos considerar que los retos de una política y de un derecho ambiental vienen aunados a un análisis y una práctica totalmente transversal del principio de sostenibilidad con el análisis conjunto de pilares fundamentales como son los aspectos económicos, políticos, sociales/ culturales y los ecológicos, tomando principios éticos como soporte de los mismos, bases que deben ser analizadas transversalmente con una serie de elementos como población, organización social, tecnología, territorio y aspiraciones sociales. Este análisis dinámico otorgado por los estudiosos de la problemática ambiental, ya antes mencionados, es de característica totalmente transversal, desde los pilares de acción hasta cada realidad perteneciente a cada uno de los factores listados, lo que permite el entendimiento real de cada problemática y el ataque de raíz a cada componente, obteniendo como resultados indicadores positivos no solo de resultados sino de real impacto en la vida de cada grupo poblacional existente en el Perú y al mismo tiempo de manera global, de todo el Perú como una unidad.

La aplicación del principio de sustentabilidad necesita claramente de la aplicación transversal de cada elemento en mención. Como señala Otis Ducan, sólo se logrará la sustentabilidad con el equilibrio dinámico de los elementos ya referidos ${ }^{183}$. Es decir, cada vez que se estudie una posible política ambiental deberá analizarse la satisfacción de aquellos elementos.

Finalmente, podemos sintetizar que el éxito de un política ambiental está basada en esta especial dialéctica y sinergia de los elementos en mención, de modo transversal y sostenible, sin dejar de considerar su característica colectiva, donde el trabajo de todos implica la real efectividad y eficiencia de los proyectos, por lo que es necesaria la aplicación de los principios de información y participación ciudadana sostenidos por una democracia que permita la representación de los ciudadanos, el respeto a los derechos humanos, a la separación de poderes, al estado de derecho, a las instituciones y al orden jurídico establecido. 